\title{
Verbal and Nonverbal Reactions of Preschoolers During Unsolvable Task
}

\author{
${ }^{1}$ Marija Letica, ${ }^{2}$ Lara Cakić, ${ }^{3}$ Vedrana Kuti \\ ${ }^{1}$ Faculty od Education, J.J. Strossmayer University of Osijek, Croatia, ${ }^{2}$ Faculty,od Education, J.J. \\ Strossmayer University of Osijek, Croatia, ${ }^{3}$ Faculty od Education, J.J. Strossmayer University of Osijek, Croatia
}

\begin{abstract}
.
Current research examined the relationship between mothers and teachers assessments about levels of anxiety-depressive symptoms of preschool children and the correlation between children level of anxiety-depressive symptoms and verbal and nonverbal reactions of children during solving unsolvable task. Anxious/depressed problems are measured by The Child Behavior Checklist (TRF, CBCL - Achenbach, 1991). Verbal and nonverbal reactions were recorded and then coded. Research included 53 children (31 boys and 22 girls who were five to seven years old), their mothers and their teachers $(\mathrm{N}=10)$. Results show statistically significant negative relationship between verbal reactions focused to the task and nonverbal reactions focused of the task. Results show no statistically significant relationship between assessments of mothers and assessments of teachers about level of anxiety-depressive symptoms. Positive relation between assessments of teacher and verbal reactions focused of the task is statistically significant as well as correlation between verbal reactions focused to the task and nonverbal reactions focused of the task.
\end{abstract}

Key words: verbal reactions, nonverbal reactions, preschoolers, anxiety, unsolvable task 